\title{
DYNAMIC LINE RATING AND AMPACITY FORECASTING AS THE KEYS TO OPTIMISE POWER LINE ASSETS WITH THE INTEGRATION OF RES. THE EUROPEAN PROJECT TWENTIES DEMONSTRATION INSIDE CENTRAL WESTERN EUROPE
}

\author{
Huu-Minh NGUYEN \\ University of Liège - Belgium \\ HM.Nguyen@ulg.ac.be
}

\author{
Jean-Louis LILIEN \\ University of Liège - Belgium \\ JL.Lilien@ulg.ac.be
}

\author{
Peter SCHELL \\ Ampacimon s.a. - Belgium \\ Peter.Schell@ampacimon.com
}

\begin{abstract}
The general increase of Distributed \& intermittent renewable Generation is pushing TSOs and DSOs to find innovative solutions to increase the capacity of their networks. Dynamic Line Rating (DLR) has proven to be a very promising solution to cope with this issue. However, few experiments so far have addressed ampacity forecasting, which is critical to allow the integration of DLR in the electricity market processes. To tackle this gap, we developed an algorithm based on real-time DLR measurements and weather forecasts, which provides ampacity forecasts up to two days ahead. This paper describes the results of the EU-funded TWENTIES Demo led in Belgium on two $150 \mathrm{kV}$ lines. Currently, the DLR system used provides a two-day ahead ampacity forecast with more than 10\% average gain over static rating (dynamic gain forecast varying roughly between $0 \%$ and $30 \%$ ) with $98 \%$ confidence. Operational gains allowed by this increase of flexibility are still being assessed.
\end{abstract}

\section{INTRODUCTION}

Wind farms and PV development have continuously grown over the past years. In Europe only, wind farm installed capacity was $90 \mathrm{GW}$ in 2011 with more than $10 \%$ annual growth [1]. This change in the production portfolio generates very significant needs for additional transport \& distribution capacity at all levels of the network. It is more and more obvious that replacing, uprating and building the required new electrical power lines $(50000 \mathrm{~km}$ in Europe) [2] will become the main bottleneck to reach the EU 20-2020 objectives. Therefore it has become mandatory to develop new approaches to increase the efficiency of the existing network assets in a secure way and deliver the required capacity in a timely and economically viable way.

Intermittent \& distributed generation significantly changes the characteristics of the power flows in the various networks, increasing the need for capacity but at the same time reducing the line usage factor (MWh transported per MW of transmission/distribution capacity) of the network assets due to the increased volatility and variability of the flow patterns. One of the solutions to adapt the system to these new constraints is Dynamic Line Rating (DLR), which allows TSOs and DSOs to monitor their existing assets in real-time and significantly increase the dynamic line capacity, also known as ampacity, over the traditionally used static ratings.
Moreover, Dynamic Line Rating of overhead lines combined with Active Network Management have proven to be very promising in this frame [3], as a strong positive correlation between wind farm generation and an increase of nearby transmission \& distribution lines ampacity have been shown. Extra line capacity is thus available when required, and this without waiting for infrastructure reinforcements/extensions that often lead to both long delays and cumbersome costs. The overall optimum efficiency is obtained when DLR is combined with the possibility to adapt/curtail generation. When DLR is used, allowing a small percentage of curtailment significantly increases the amount of renewable generation that can be connected to the existing network, and thus gives an optimal solution for all the concerned actors: TSO, DSO and wind farm owners and investors.

However, up to now, few experiments addressed ampacity forecasting, which is of prime interest for TSOs, DSOs and the whole electricity market. Indeed most decisions regarding the operation of the network are taken either one day ahead or 2 days ahead, such as the capacity nominations (NTC) for the cross-border energy markets. Some papers deal with short-time prediction (within one hour) [4], while another address 24-hour ampacity forecast solely based on weather forecast [5].

But still, these promising tentative results are not satisfactory yet, as the objective of 2-day ahead ampacity forecast is not reached, and as prediction interval is highly dependent on weather forecast accuracy.

Therefore, the University of Liège (ULg), Belgium, developed an algorithm based on real-time DLR measurements and weather forecasts to provide two-day ahead ampacity forecasts with controllable prediction interval.

The following sections describe the ampacity calculation method, and the high sensitivity of dynamic rating w.r.t. wind speed. The test campaign and results obtained are then presented, followed by the conclusion.

\section{AMPACITY CALCULATION METHOD}

The ampacity of a conductor is that maximal constant electrical current which will meet the design, security and safety criteria (i.e. electrical clearance) of a particular line on which the conductor is used [6] (Fig. 1 and 2). 


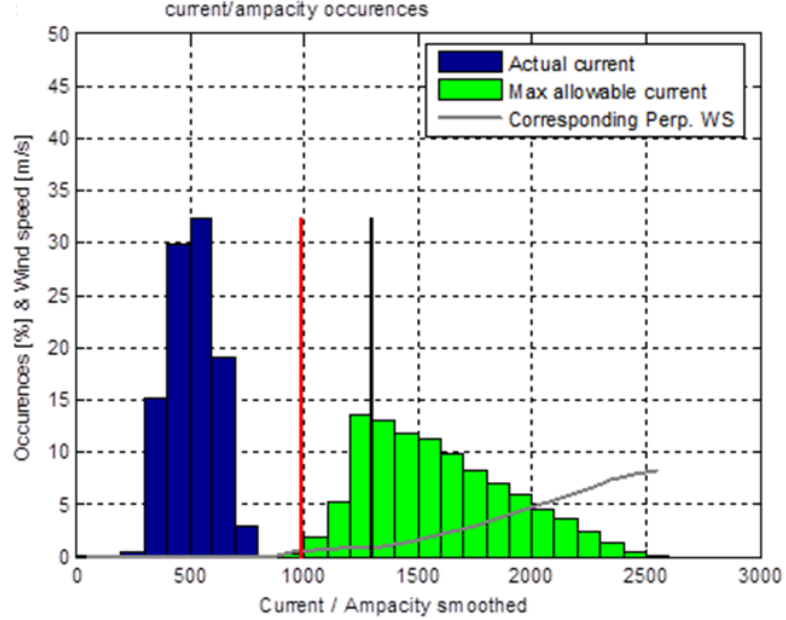

Fig. 1: typical histogram of power line loading. Static rating is 1000 A. Actual loading on the left hand part (under normal operating conditions) and available ampacity, on the right-hand part, with a trend curve giving the corresponding effective wind speed (same scale as occurrences but in $\mathrm{m} / \mathrm{s}$ ).

The methods to evaluate ampacity from data are explained in many books [7] and technical brochures from international organizations, such as CIGRE publications [6, 8], which use weather data as locally measured or simulated following international recommendations as explained, for example, in CIGRE [8] or IEEE [9].

\section{AMPACITY HIGH DEPENDENCY ON WIND SPEED}

The wind speed has a dramatic impact on power line ampacity as it is the main variable responsible for cooling down the conductor, and hence for the sag value.

But wind speed measurement is tricky for various reasons. First, it is not stationary as wind speed can vary significantly within minutes. Second, it also varies along the span (spatial coherence): wind vortices have a typical average size of several tens of meters [10]. Therefore, a typical span length of several hundreds of meters is subject to a variable wind speed along its length. Third, the wind speed also varies greatly vertically, as the conductor is fastened within the boundary layer, and as the span's lowest point is generally about 10 meters over the ground. The wind speed may also vary due to local effects, such as screening from trees or buildings, altitude of the conductor which may change in a single span of more than 15 meters if only the sag is considered, but which may also be subject to difference of levels between end points of a span. Such a difference in altitude near the ground may have huge effects as the conductor lies in an air layer located in the boundary conditions of wind speed variation due to the ground proximity.

Therefore, a single-spot measurement does not allow computing the global effect of the wind over the whole span.
All of these factors are particularly important for low wind speeds (typically for wind speeds component perpendicular to the conductor axis lower than $3 \mathrm{~m} / \mathrm{s}$ ), which have the most dramatic impact on ampacity. Similarly, a single-spot measurement of "effective incident radiation" does not allow to compute the global effect of the combined effect of sun and albedo over the whole span.

These measurement issues can be dealt with in real-time using an appropriate DLR device, which directly catches the effects of these weather variables on the conductor instead of measuring them and trying to estimate the effects based on the theoretical thermal model applied to the conductor. This direct approach guarantees at all times that the conductor is operated within safe limits.

Actually, the smart sensor installed on the conductor, as part of the Twenties demo, is able to produce a certified sag (without any external data) and corresponding ampacity in real time and up to a few hours ahead (up to 4 hours), see Fig2.

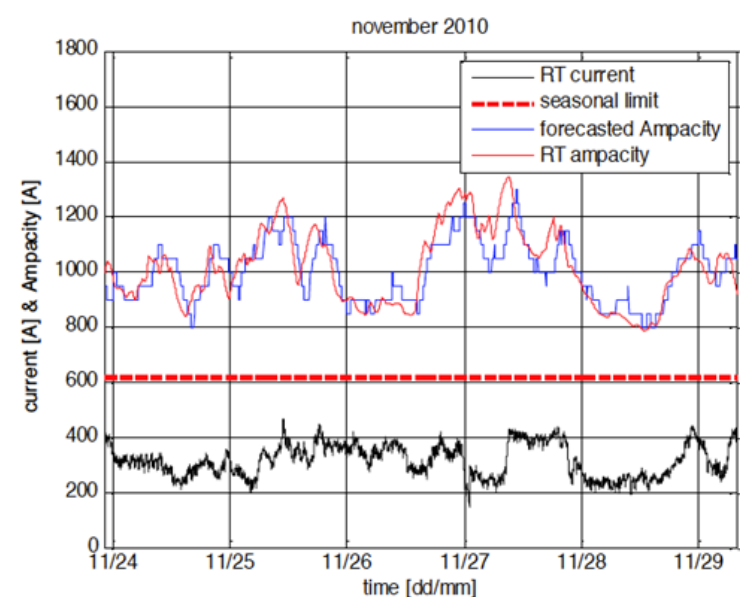

Fig. 2: One week of power line loading (bottom curve) as typically used under normal operating conditions. Static rating at 600 A (horizontal dotted line), DLR and its one hour ahead forecast on the upper curves.

But forecasting ampacity beyond a horizon of a few hours is a challenge as no field measurement is available at that moment, and as wind forecast deduced from Numerical Weather Prediction models cannot catch the local aspects on site, especially for low wind speeds blowing below $3 \mathrm{~m} / \mathrm{s}$. An experiment was needed to develop and validate ampacity forecast algorithms. We therefore set up a measurement and forecast campaign which is part of the TWENTIES project Netflex DEMO. This is described in the next section.

\section{TEST CAMPAIGN}

An original experiment has been carried out in the EU funded TWENTIES Project, Netflex DEMO: a project which started in 2010 and finished in March 2013 with a Demo for Central Western Europe (CWE) supervised by CORESO. 
This DEMO shows how DLR can be combined with WAMS (Wide Area Measurement Systems using Phasor Measurement Units) and PSTs (Phase Shift Transformers) to control and optimize line loads in an operational way in the CWE region.

This paper will exclusively focus on the DLR aspect of the experiment. Two $150 \mathrm{kV}$ lines in the North of Belgium were equipped with 10 Ampacimon $^{\circledR}$ sensors (Fig.3), these are two parallel circuits installed on the same towers in the Belgian Network. The lines are located near the North Sea coast where offshore wind farms are installed.

In parallel some meteorological data were gathered:

- $\quad$ some existing remote (10 $\mathrm{km}$ away from the line) weather stations were recorded, those weather variables were used as initial inputs together with sag measurements to compute a conservative ampacity in real-time;

- meteo forecasting (up to 60 hours ahead with a new forecast every 6 hours) was developed using world wide GFS (Global Forecast System) and local WRF (Weather Research and Forecasting) analysis;

- the postprocessing of the meteo forecast was done using different local data and/or statistical tools with reinforcement learning based on Ampacimon sensor outputs in real-time to provide the ampacity forecast
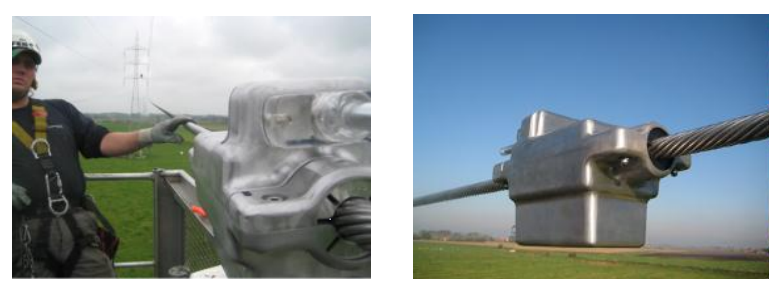

Fig 3: Installation of the smart grids sensor Ampacimon on a $150 \mathrm{kV}$ line in the north of Belgium, near the North Sea.

\section{RESULTS}

Remarkable results could be attained as shown in Fig. 4, 5 : Solely weather-based ampacity forecast provides the trend for the following days, but fails to give reliable ampacity forecasts, mainly due to wind speed forecast uncertainties. In contrast, the ampacity forecast algorithm we developed provides a safe forecast, though with less gain than the actual ampacity measured in real-time. In the case shown, the forecast average gain was roughly $15 \%$ vs. $50 \%$ for the actual average gain in real-time. Nonetheless, this method has the advantage to give some control on the prediction interval, depending on the safety margin needed. Here, the chosen value was $98 \%$ confidence (Fig 5).

This gain-confidence tradeoff on forecast can further be used by TSOs and DSOs to make the best decision. This implies a more probabilistic approach to network operational management which is not implemented in the procedures yet, but we firmly believe this kind of approach can't be avoided in the future for further integration of RES.
Based on the DEMO outputs, some virtual extrapolations have been examined on passed contingency events in the CWE region to evaluate the potential of this DLR solution to solve these contingencies. Results are still being assessed, but the first analysed cases already show more than $10 \%$ forecast gain over the static rating for the whole day of the analysed contingency cases. PSTs and WAMS can be further used to add more flexibility. This gain would have relieved these specific congestion cases, avoiding topological and/or costly redispatch actions. Furthermore, capacity increase has a general favourable impact on market coupling, thus increasing overall welfare in EU.

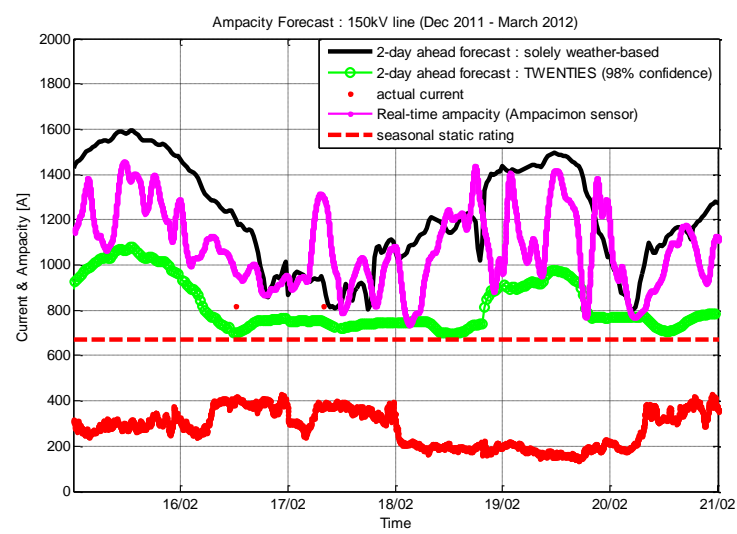

Fig. 4 : (i) actual current (red), (ii) real-time ampacity (pink), (iii) solely weather based two-day ahead forecast (black) only gives a general trend, (iv) the ULg algorithm developed guarantee a safe ampacity forecast



150kV line from Dec 2011-March 2012

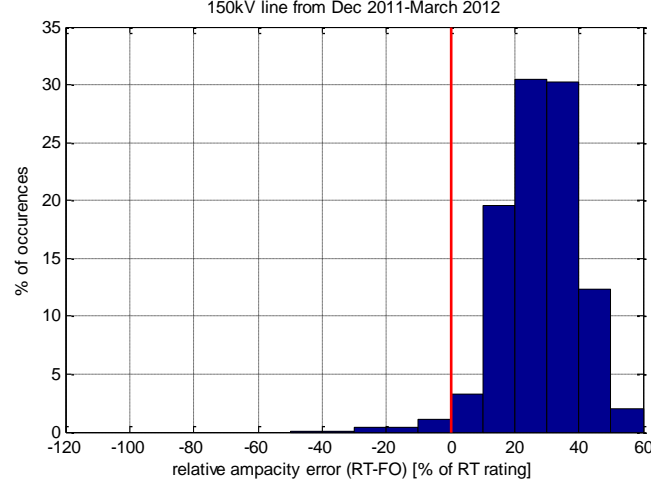

Fig. 5: Relative ampacity error between real-time and two-day ahead forecast ampacity ; top : solely weather based forecast, bottom : ULg forecast algorithm (98\% confidence) 


\section{CONCLUSION}

Increasing RES penetration requires innovative solutions to maximise the capacity of the existing network. Dynamic Line Rating already helps TSOs and DSOs better manage new constraints in their networks in real-time, and intra-day (a few hours ahead) by increasing line rating limits, but significant additional added value lies in the ability to forecast ampacity up to two days ahead.

An original experiment has thus been performed since 2010 on the Belgian network, with virtual extrapolations on Central Western Europe network supervised by CORESO. An ampacity forecast algorithm has been developed to forecast ampacity up to two-day ahead from real-time DLR measurements and weather forecast.

It succeeded in its purpose because weather forecast were considered in a new way with a clear emphasis on low wind speed period forecasting owing to online sensors used as tuning data. Reinforcement learning tools coupled with stochastic methods finalize the post-processing step, providing a controllable prediction interval.

On the studied line, more than $10 \%$ average gain over static rating could be obtained (dynamic forecast gain roughly varying between $0 \%$ and $30 \%$ ) with $98 \%$ confidence for a two-day ahead ampacity forecast.

No doubt that such tool will help future development in dynamic management of power system adding a new variable: the fact that a power line has a dynamic rating varying between $98 \%$ and about $200 \%$ of the actually used static rating! Obviously some check on stability and assets need to be done before using the upper limit. But most of the time $120 \%$ is far enough to solve most of the contingencies.

Dynamic Line Rating is thus definitely part of the global solution to this issue.

Impact on power system management will be huge. We are at the dawn of a new era which will allow investments to focus on where it is needed most (offshore networks,...), will help congestion management, will limit power redispatch and will avoid or at least minimize wind farm curtailment, by providing the network managers with a safe and reliable solution to operate their existing overhead lines at maximum efficiency.

Those results clearly show how smart devices and software can be used to optimize TSO's and DSO's existing assets and investments in the paradigm shift we're facing today.

\section{ACKNOWLEDGMENT}

These developments have been made possible owing to EU support inside the TWENTIES project, which is part of the seventh framework program (www.twenties-project.eu). The partners of NETFLEX demo inside Twenties have to be thanked owing to their major contribution:

ELIA, coordinator (Belgian TSO), CORESO (European supervisor of the network), Siemens Belgium (hardware integration), ULg, KUL and ULB (three Belgian universities), Ampacimon SA (sensor manufacturer as well as global integrator of DLR part inside Coreso). In particular the collaboration between ULg power system department (authors) and ULg topo-climatology department
(Pr. M. Erpicum and S. Doutreloup) was particularly useful.

\section{REFERENCES}

[1] ENTSO-E, 2012, Statistical Yearbook 2011, Secretariat of ENTSO-E, Berlin, Germany, 10

[2] ENTSO-E, 2012, 10-Year Network Development Plan 2012, ENTSOE AISBL, Brussels, Belgium, 62.

[3] P.Schell, J.-L. Lilien, H.-M. Nguyen and al., 2012, "Large penetration of distributed productions: dynamic line rating and flexible generation, a must regarding investment strategy and network reliability", CIRED Workshop. Lisbon, IET, paper 280.

[4] M. Musavi and al., 2011, "Overhead conductor dynamic thermal rating measurement and prediction", International Conference on Smart Measurements for Future Grids (SMFG), IEEE, 135-138.

[5] A. Babs, 2011, "Weather-based and conductor state measurement methods applied for dynamic line rating forecasting", International Conference on Advanced Power System Automation and Protection., IEEE, 762-765.

[6] "Guide for selection of weather parameters for bare overhead conductor ratings", 2006, Cigre technical Brochure No. 299, Study Committee B2, 10.

[7] Kiessling F., Nefzger P., and al., 2003, Overhead Power Lines. Planning, Design and Construction, Springer, Berlin, Germany.

[8] "Thermal behaviour of overhead conductors", 2002, Cigre Technical brochure No. 207, Study Committee B2.

[9] IEEE Standard 738-2006. "IEEE Standard for Calculating the Current-Temperature of Bare Overhead Conductors", IEEE Power Engineering Society, 2006.

[10] Emil Simiu, Robert Scanlan, 1996, Wind Effects On Structures, Third Edition, John Wiley and Sons.

\section{AUTHORS}

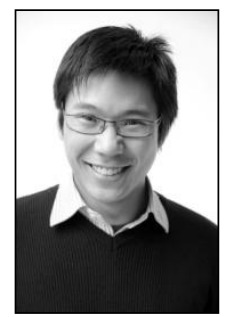

NGUYEN Huu-Minh graduated from University of Liège, Belgium, as a physics engineer. He has been working as a teaching assistant and $\mathrm{PhD}$ student for 4 years in the Transmission and Distribution of Electrical Energy Unit at the Montefiore Institute of Technology, University of Liège, Belgium. His main activity has been to deal with real-time High-voltage overhead lines maximum carrying-current capacity (known as "ampacity"). He has been working on the development and implementation of algorithms for this purpose at the University of Liège, and is now working on 48-hour ahead ampacity prediction, using machine learning and statistic algorithms. He is actually supported by European project TWENTIES

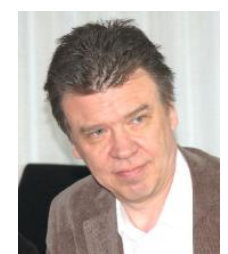

Professor J. L. Lilien, PhD., is the head of the unit Transmission and Distribution of Electrical Energy at the Montefiore Institute of Technology, University of Liège, Belgium. He has over 30 years experience solving the electrical and mechanical engineering problems of power systems. His work involves analysis of problems in "cable dynamics" in general and on overhead power lines in particular. His major activities were devoted to (i) vibrations on transmission lines, in particular galloping, including its control (ii) large movements of cables, like short-circuit (both in substations and power lines), (iii) health monitoring of power lines (sag and vibrations) and last, but not least (iv) low-frequency electric and magnetic field effects on human beings. He is a longtime active member of IEEE and CIGRE, where he has served as convenor of several task forces of CIGRE study committee B2, "overhead lines" and B3 "susbstations". He has published over 100 technical papers in peer reviewed publications. He is the initiator and organiser from 1995, of the CABLE DYNAMICS conference.



Peter Schell was born in Brussels, Belgium, on July 15, 1970. He studied at the University of Stuttgart, Germany and graduated in 1995 as Dipl. Ing. Luft- und Raumfahrttechnik. His employment experience includes large-scale IT projects at amongst other Deutsche Bahn Cargo and the French distributor Auchan. After that he specialized in Utilities and worked for more then 10 years for different Belgian Utilities helping them adapt to the liberalized market and prepare for the roll-out of smart meters. Since February 2011 he is the General Manager of Ampacimon. 